\title{
Uncertain parameter numerical model updating according to variable modal test data in application of large composite fuselage panel
}

\author{
Marcin Luczak, Antonio Vecchio, Bart Peeters, Ludo Gielen and Herman Van der Auweraer* \\ LMS International, Interleuvenlaan 68, B-3001 Leuven, Belgium
}

\begin{abstract}
This paper presents a novel approach in the field of experimental and numerical investigation of mechanical properties of composite structures. It takes into account test data variability resulting from structural dynamic properties measurement and uses them to quantify uncertainties in model parameters updating. The main goal of the conducted research is to investigate the dynamic properties of fibre reinforced composite structures. Non-destructive experimental and numerical simulation methods are used hereto. In the experimental part, different test configurations were taken into account. The excitation was performed by means of random and harmonic, single and multi point stimuli while the response measurement was done through contact and non-contact acceleration, velocity and dynamic strain sensing. The test results are applied in two ways: for the structural identification of the object and for non-deterministic updating of the numerical model according to a range of experimental models obtained from test. The sources of the test data variabilities were related to the excitation and measurement technique applied for the investigated object. Non - deterministic model updating and verification \& validation included uncertainties of its parameters by means of interval and stochastic methods. A number of variable test modal models were statistically assessed to investigate impact of variability source onto modal model parameters. The presented research was conducted in the context of the FP6 Marie Curie project UNVICO-2.
\end{abstract}

Keywords: Modal testing, uncertainty, updating, strain

\section{Introduction}

The usefulness of structural dynamics test and analysis results for solving noise and vibration problems or for performing a design assessment or a design optimization, depends largely on the confidence that one can have in these results. In other words, the results must be characteristic for the actual problem (and not be the result of testing artifacts) and the models must be representative for the actual behavior of the investigated structure (s). This problem of modeling uncertainty has consequently been the subject of major research efforts [1-4]. Essentially, two types of problems are distinguished: (1) the test and modeling data are subject to experimentation and analysis errors and (2) the tested (or modeled) structure is not representative for the actual structure.

The first problem is this of experimentation and analysis uncertainty [5,6]. The "true" test result can in principle never be achieved. The level of the uncertainty associated with the test result is however not easy to quantify. A multitude of totally different causes may be at the origin of major bias and/or variance errors in the analysis [7-10]. Adequate testing and analysis procedures may reduce (at least some of) these errors, but proper overall uncertainty quantification is hard to issue. Also in building numerical simulation models (e.g. based on the Finite Element

\footnotetext{
*Corresponding author: E-mail: Herman.vanderauweraer@1msintl.com.
} 
approach), uncertainty is introduced by discretization effects, through imperfectly known material, geometry or loading parameters, or through uncertainty in the applicable model formulations [11-13]. Various approaches based on stochastic or possibilistic methodologies have been developed to address this [11-17].

The second problem is this of product variability [18], introducing changes in the structural dynamics characteristics because of differences in material, geometric, manufacturing or even operational use (loading, temperature ... ) parameters when compared to the "nominal" case. It is important to have at least an idea on the magnitude of these changes and their impact on the final product behavior when assessing and optimizing the design based on an "ideal" product model. While statistical product testing may reveal such performance spread, this is in general very hard to realize.

A third (and often neglected) potential problem with the significance of the analysis results can originate from a violation of the assumptions used to model the structure. One of the most prominent examples is the effect of nonlinear structural behavior on a linear (e.g. modal) model identification process.

The review of relevant scientific literature in the field points out that in most practical engineering applications there is a serious lack of experimental data that would enable a correct quantification and characterization of stochastic system properties [1-17]. The current projects work introduces a novel approach in the field of experimental and numerical investigation of mechanical properties of composite structures. It takes into account test data variability resulting from structural dynamic properties measurement and uses them to quantify uncertainties in model parameters updating.

\section{Methodology}

The starting point of an experimental structural dynamics assessment is modal analysis testing. The corresponding modal model results are considered to be a deterministic system description, which can be used for multiple applications, ranging from a mere verification of the fulfillment of the design criteria, to the validation and updating of CAE models and the integration in hybrid system models. In reality, the modal results are just an estimation of the model parameters based on a series of input-output or output-only tests and hence subject to all related testing and modeling errors. These data errors can be just stochastic disturbances on the input/output data, but can also be caused by invalid model assumptions or data processing effects. Some of the main sources of errors are: sensor location and orientation errors, test set-up loading and constraining effects, sensor loading effects on the test structure, sensor calibration and data conversion errors, disturbance and distortion in the test data measurement chain, signal processing errors and model estimation errors.

Just repeating a test and looking into test statistics will not allow the majority of these sources of uncertainty to be identified. Good practice is hence to repeat tests in slightly different test configurations, by adopting different excitation or sensing configurations and evaluate resulting model differences to assess the robustness of the test procedure and confidence in the test results. In the present study, two kinds of such test variation are executed. The first is by analyzing the variation of global system parameters (resonance frequencies, damping values) as a function of changes in the instrumentation (type of response sensors - contact vs. non-contact-, number and orientation of shakers, excitation signal). The second is by analyzing the variation of global system parameters when splitting up the measurement in several sections ("patches"). The corresponding inter-patch variations which give cause to inconsistencies when attempting a global analysis on the complete data set [19]. While from a rigorous statistical point of view, such analysis may not yet provide the means to derive stochastic bounds on results, the related spread on the data and the models is one which is clearly representative for the daily measurement practice.

In order to characterize the variability of the results, one can obviously look into the distribution of the modal parameters as a function of test condition. This requires the adoption of very reliable and robust modal parameter estimation methods that allows to easily identify modal parameters from complex multi-mode datasets. An example is the discrete frequency domain rational polynomial model technique, referred to as PolyMAX [20]. Its fast and robust identification process allows the application to analyzing large and multiple datasets, making variability and statistical analysis possible.

The search for unique qualifiers for the variability of a multi-test data set has led to the definition of a new parameter named Variability Level which characterizes a particular structure in view of its variability. It is calculated as the mean value of a standard deviation of the modified frequency and damping. 

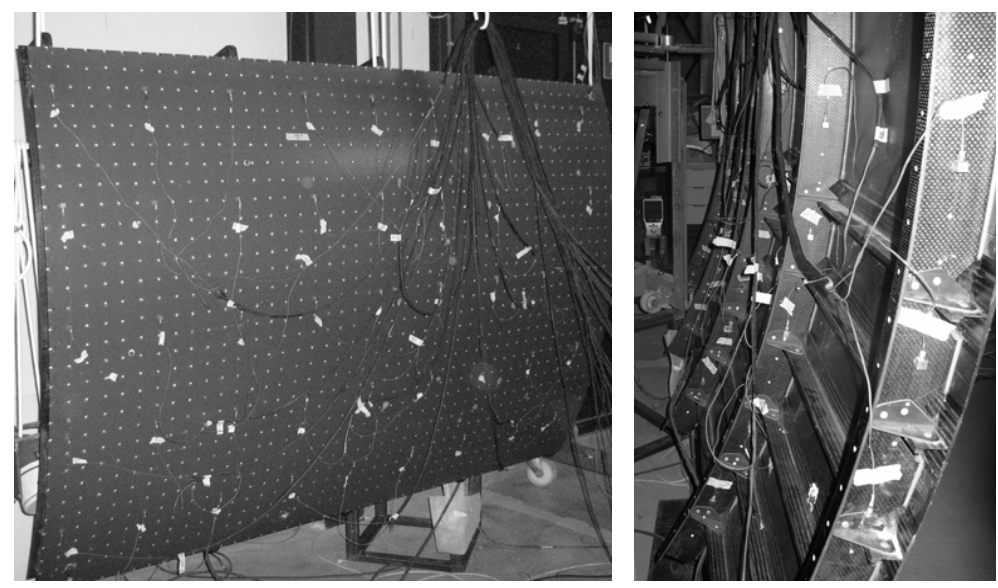

Fig. 1. Front (left) and rear (right) view of the composite fuselage panel.

Modified dimensionless frequency values are calculated according to the:

$$
f_{\text {mod }}=\frac{(f-\hat{f})}{f}
$$

Mean value of the standard deviation of the modified frequency is Variability Level.

Presented measure of the variability is also applicable to different cases than presented in this paper. In case of measuring the modal parameters of the nominally identical structures it can be used to assess the non-repeatability of the manufacture process. This is especially valid for the composite material structures which suffer from scatter of the design parameters resulting from the limitations of the manufacturing technology. It also allows assessing the influence of the environmental factors like temperature or humidity on the obtained results of measurement of the same structure in different weather conditions.

The final use of many experimental models is to validate and update numerical models, precisely because the latter are subject to many sources of modeling uncertainty [21,22]. However, when the experimental models themselves are subject to uncertainty, this introduces another level of uncertainty. In the present paper, a standard model updating process is adopted, essentially validating the impact of the experimental uncertainty on the numerical updating results. However, more advanced numerical updating methodologies exist which explicitly take into account the uncertainty in the test data to come to a stochastically-optimized updating procedure [23-26].

In the present paper, this procedure will be applied to a specific, industrially relevant case study. The subsequent steps will include the definition of multiple experiments, the analysis and assessment of the uncertainty in the data, the extraction of the modal parameters and the use in a model updating procedure.

\section{Test structure and measurement set-up}

The object of the investigation is an advanced fuselage panel presented in Fig. 1. It is composed of five vertically oriented frames and seven horizontal stiffening stingers on which a three-section skin is assembled. Each of three main subcomponents is made of Carbon Fibre Reinforced Plastics. They are fastened by means of glue and metal joints. Dimension of the investigated panel are: Height $\approx 1700 \mathrm{~mm}$, Width $\approx 2234 \mathrm{~mm}$, Thickness of the skin top section 28 plies, middle section 15 plies, bottom section 14 plies $0.147 \mathrm{~mm}$ each and the radius of the curvature $\approx$ $1975 \mathrm{~mm}$. Weight of the structure is $33.25 \mathrm{~kg}$.

In test campaign the following measurement and analysis tools were used:

- Panel supported by a 3 rubber cords for providing free-free boundary condition, Fig. 1;

- 2 electromagnetic shakers, amplifiers, with impedance heads incorporating acceleration and force sensor in the same housing to measure driving point FRF's; 


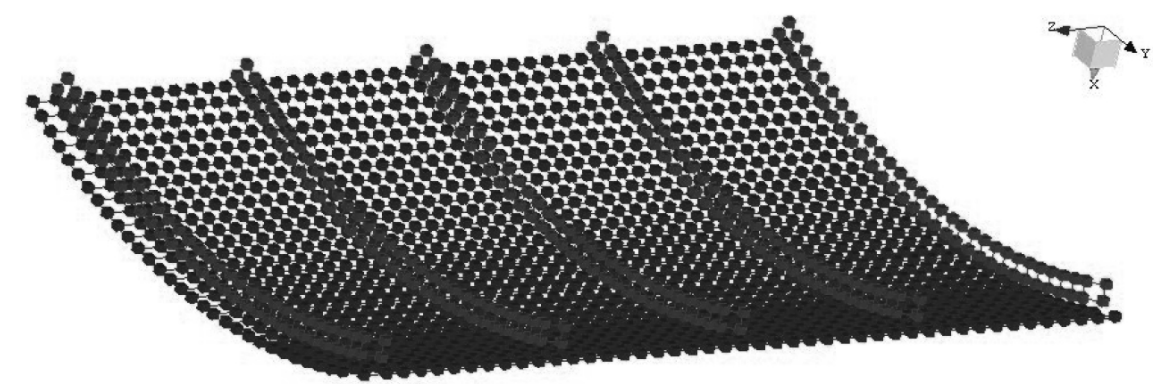

Fig. 2. Measurement grid for skin (1395 points) and frames (310 points) in Cartesian coordinate system.

- 45 uniaxial modal piezoelectric accelerometers PCB 333B32, applied to both panel and frames;

- Scanning Laser Vibrometer OFV3001S;

- Microflown probes: PU-mini NT0712-44 and USP-mini UT0608-01;

- Dynamics strain sensors;

- 64 channels in frontend LMS SCADAS III with computer with a Test. Lab acquisition and analysis suite.

The geometry definition for skin and frames is shown in Fig. 2.

The large number of measurement points is required for correlation analysis performed between the experimental and Finite Element models. Traditionally the FE model contains much more nodes in comparison to the experimental one. This poses the need for the FE model reduction or expansion of the experimental model. Both techniques suffer from systematic errors which introduce higher uncertainty into model updating procedure. The high number of the measurement points reduces the influence of this error.

\section{Experimental modal analysis}

\subsection{Overview of tests and models}

Experimental modal analysis (EMA) is used to establish a structural dynamics model of the structure under test. While in principle such model is unique, in practice the estimated modal models differ as a function of number of shakers, excitation method, measurement directions and measurement technique used.

In the present case study, three different measurement approaches were used, one based on the use of piezoelectric sensors, one based on the use of a laser Doppler vibrometer and one base on the use of acoustic particle velocity sensors (PU-probe from Micoflown). For the piezoelectric sensors, accelerations response was measured. For the laser Doppler vibrometer and Microflown probes the velocity response was measured. Furthermore, different excitation configurations were evaluated, using respectively one shaker, two shakers (out-of-plane) and two shakers with oblique orientation. Burst random as well as sine excitation were employed. For all models, the shaker input force was used as a reference.

Particular care had to be taken with the accelerometer measurements as the lightweight nature of the structure prohibited the application of significant number of sensors simultaneously. Therefore the measurements were conducted by means of consecutive "patches", each containing a limited set of measurement points and leading to a "local" dataset. The accelerometer measurements were performed direction by direction, which allowed separate analysis for out-of-plane data only and for a full 3D dataset.

To execute these test objectives, a number of different test models were established. Five global modal models were estimated from out-of-plane direction measurements. Two models were established containing - for one configuration - the response data in the other two directions and for two shaker orientations (out-of-plane and oblique). This resulted in the following datasets:

M1: 1 shaker burst random excitation X direction measurement, 1 impedance head, 54 accelerometers and 38 sets of location of the sensors were applied to cover all the measurement grid, 


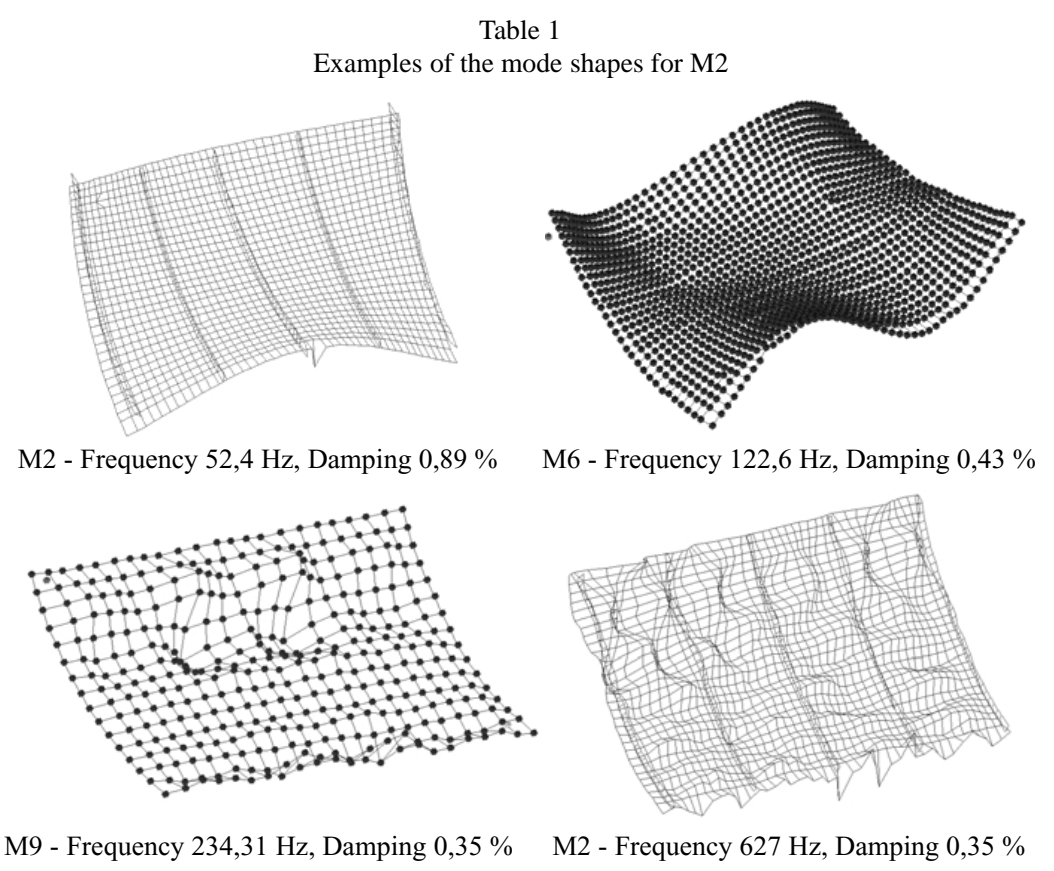

M2: 2 shakers burst random excitation $X$ direction measurement, 2 impedance heads, 45 accelerometers and 43 sets of location of the sensors were applied,

M3: 2 shakers burst random excitation (out-of-plane shaker orientation) X,Y,Z direction measurement, 2 impedance heads, 45 accelerometers and 43 sets of location of the sensors were applied,

M4: 2 shakers burst random excitation (oblique shaker orientation) X,Y,Z direction measurement, 2 impedance heads, 45 accelerometers and 43 sets of location of the sensors were applied,

M5: 2 shakers sweep sine excitation X direction measurement, 2 impedance heads, 45 accelerometers and 43 sets of location of the sensors were applied.

M6: 2 shakers burst random excitation X direction measurement, 2 impedance heads, 1 Scanning Laser Vibrometer Optical Sensor

M9: 2 shakers burst random excitation $\mathrm{X}$ direction measurement, 2 impedance heads, 2 microflown probes and 192 sets of location of the measurement points

(M7 and M8 consisted of datasets not further discussed in this paper)

More details on the various experimental procedures, can be found in [27]

\subsection{Analysis results}

In order to extract the modal parameters, the frequency domain rational polynomial method "PolyMAX" [20] was used as it allowed very reliable, unequivocal and fast analysis of the large number of datasets.

In total 51 local modal models were estimated, related to the locations of the various sets of sensors. Applying the multi-run modal analysis approach [19] the 51 local models were merged into eight global modal models. The 51 modal models (samples) constitutes a significant collection of experimental data which is sufficient for quantifying uncertainty by means of the statistical methods

As an example of the modal analysis results, some typical results are shown below. Tables 1 and 2 present a number of mode shapes obtained for the out-of-plane responses with different measurement techniques

Results from global models estimated from XYZ direction measurement (M3, M4) are shown in Table 2.

For the XYZ models it can be observed that the transverse displacements in in-plane direction were measured for the frames. 
Table 2

Examples of the mode shapes for M3

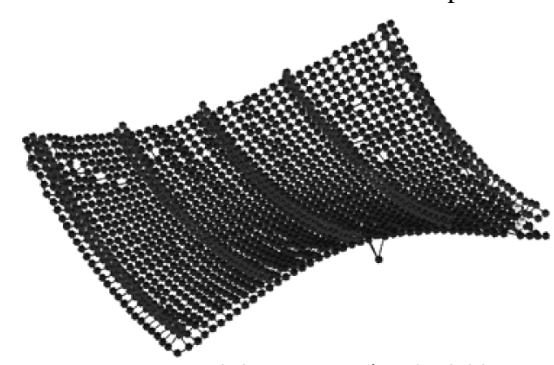

Frequency $52,8 \mathrm{~Hz}$, Damping $0,53 \%$

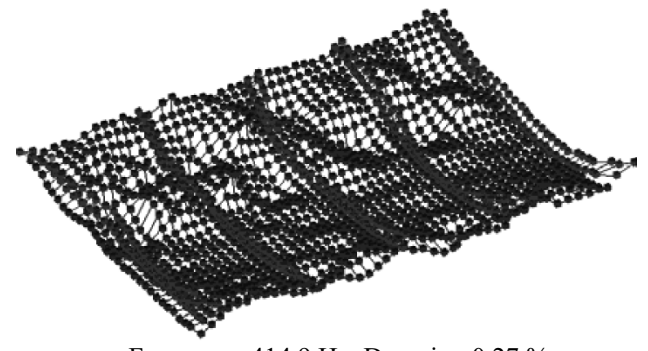

Frequency $414,9 \mathrm{~Hz}$, Damping $0,27 \%$

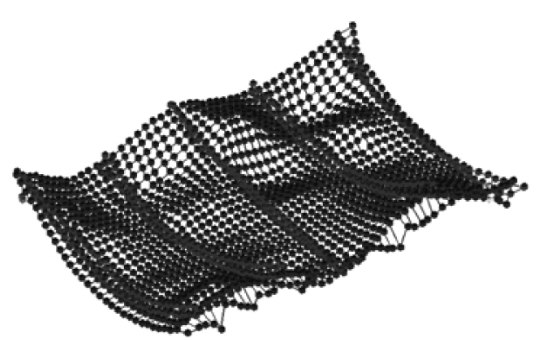

Frequency 197,8 Hz, Damping 0,5\%

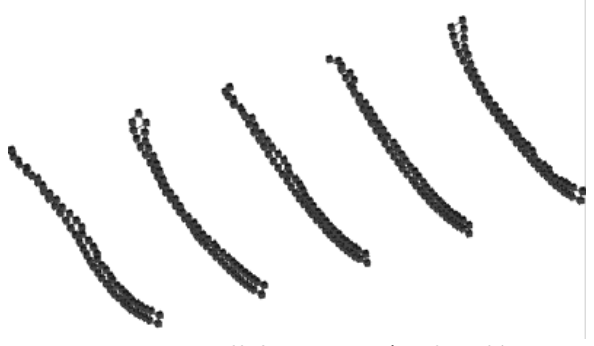

Frequency $562,8 \mathrm{~Hz}$, Damping $0,55 \%$

Table 3

Exazmples of the natural frequencies and the modal damping ratio

\begin{tabular}{|c|c|c|c|c|c|c|c|c|c|c|c|c|c|c|}
\hline \multirow[b]{2}{*}{ Mode } & \multicolumn{2}{|c|}{ M1 } & \multicolumn{2}{|c|}{ M2 } & \multicolumn{2}{|c|}{ M3 } & \multicolumn{2}{|c|}{ M4 } & \multicolumn{2}{|c|}{ M5 } & \multicolumn{2}{|c|}{ M6 } & \multicolumn{2}{|c|}{ M9 } \\
\hline & Frq, $\mathrm{Hz}$ & Dmp, \% & Frq, $\mathrm{Hz}$ & Dmp, $\%$ & Frq, $\mathrm{Hz}$ & Dmp, \% & Frq, $\mathrm{Hz}$ & Dmp, \% & Frq, $\mathrm{Hz}$ & Dmp, \% & Frq, $\mathrm{Hz}$ & Dmp, $\%$ & Frq, $\mathrm{Hz}$ & Dmp, \% \\
\hline $100-250$ & & & & & & & & & & & & & & \\
\hline 1 & 117.09 & 0.70 & 117.22 & 0.49 & 116.94 & 0.75 & 117.20 & 0.73 & 116.95 & 0.67 & 117.35 & 0.63 & 117.44 & 0.65 \\
\hline 2 & 122.37 & 0.52 & 121.94 & 0.46 & 122.16 & 0.52 & 122.24 & 0.52 & 121.91 & 0.24 & 122.62 & 0.43 & 122.55 & 0.49 \\
\hline 3 & 156.70 & 1.00 & 156.43 & 1.23 & 156.54 & 1.19 & 156.44 & 1.31 & 156.66 & 1.24 & 157.09 & 1.13 & 157.39 & 1.28 \\
\hline 4 & 159.96 & 0.45 & 159.84 & 0.52 & 159.84 & 0.52 & 159.92 & 0.50 & 159.94 & 0.37 & 160.71 & 0.44 & 160.65 & 0.45 \\
\hline 5 & 180.46 & 0.46 & 180.44 & 0.52 & 180.35 & 0.51 & 180.61 & 0.48 & 180.78 & 0.43 & 181.58 & 0.46 & 181.60 & 0.49 \\
\hline 6 & 196.29 & 0.56 & 195.42 & 0.80 & 195.44 & 0.77 & 198.11 & 0.55 & 195.67 & 0.70 & 198.75 & 0.40 & 198.72 & 0.42 \\
\hline 7 & 199.43 & 0.58 & 199.70 & 0.62 & 197.86 & 0.51 & 200.04 & 0.71 & 199.68 & 0.59 & 200.95 & 0.62 & 200.84 & 0.66 \\
\hline 8 & 211.31 & 0.44 & 210.11 & 0.64 & 210.15 & 0.54 & 210.45 & 0.50 & 210.24 & 0.63 & 210.94 & 0.37 & 210.87 & 0.51 \\
\hline 9 & 218.16 & 0.34 & 217.80 & 0.45 & 217.94 & 0.46 & 217.04 & 0.53 & 217.88 & 0.37 & 219.34 & 0.39 & 219.35 & 0.43 \\
\hline 10 & 221.69 & 0.40 & 221.00 & 0.62 & 221.13 & 0.62 & 221.19 & 0.39 & 221.57 & 0.47 & 222.44 & 0.47 & 222.41 & 0.57 \\
\hline 11 & 232.76 & 0.40 & 232.59 & 0.35 & 232.59 & 0.17 & 232.47 & 0.42 & 232.62 & 0.37 & 234.29 & 0.21 & 234.32 & 0.35 \\
\hline 12 & 243.23 & 0.38 & 242.49 & 0.49 & 242.60 & 0.46 & 243.49 & 0.56 & 242.51 & 0.46 & 244.03 & 0.41 & 244.17 & 0.44 \\
\hline 13 & 245.21 & 0.52 & 245.23 & 0.48 & 245.26 & 0.53 & 245.59 & 0.82 & 245.19 & 0.47 & 246.64 & 0.29 & 246.67 & 0.46 \\
\hline
\end{tabular}

\subsection{Comparison of the global modal models}

Modal models estimated with different excitation and measurement methods were compared to evaluate the modal data quality. Table 3 presents the comparison of frequencies of selected natural frequencies.

Within the bandwidth of interest there are all modes successfully identified for all models. Table 3 shows that analyzing all modal data in one set like it was done for the M1 and M2 is not influenced by an individual's runs data inconsistency. The estimated frequency and damping differences between model M1, M2 (all data analyzed in one set) and models M3 and M4 (partial modal models developed from an individual runs and merged into global modal model) are negligible in comparison to contact vs. non-contact measurement. Mass of the piezoceramic sensors and the cabling system added to a measured structure results in form of mass loading effect. This effect shows up in decrease of the identified natural frequency value for the same mode shape towards the higher frequencies. Visual inspection of the mode shapes presented in Tables 1 and 2 presents more local character of the mode shape towards the higher frequency value. 


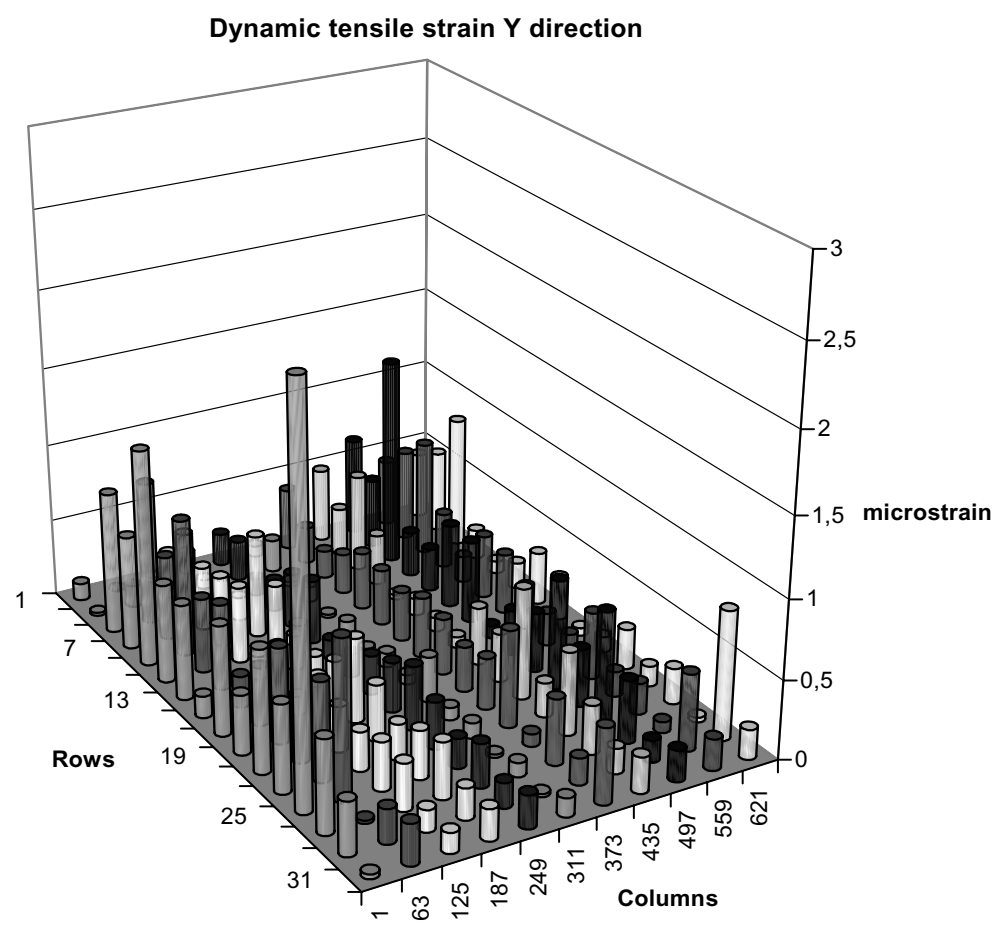

Fig. 3. Plot of the maximum values of the dynamic tensile strain.

\subsection{Dynamic strain measurement}

Next to acceleration and velocity measurement the dynamic strain was measured in selected points to observe the maximum strains. Fibers are designed to carry the tensile loads therefore this strain was main focus of interest. Figure 3 presents the graphical representation of the maximum amplitude of tensile strain in the in-plane vertical direction for the mode shape at $122 \mathrm{~Hz}$.

The distribution of the strain in particular measurement points corresponds with the maximum absolute amplitude of the displacement identified for this particular mode shape which is presented on the Fig. 4.

\section{Quantification of the variabilities in experimental data collection}

Variability assessment is of vital importance in the field of numerical model updating in presence of uncertainty on the estimated modal parameters and in the Structural Health Monitoring systems based on the observability of modal parameters alternation. Existence of large number of estimated modal models foster a statistic approach of the test data variability assessment. Dispersion of the frequency values for the 2nd mode shape is presented on Fig. 5.

This exemplary data set related to the second natural frequency is characterized in Table 4. On this plot it can be observed that there is an influence of the mass of the sensors on the measured and estimated natural frequency. For models s1-s35 the number of accelerometers attached to the structure is the same. Their additional mass attached to the investigated structure remains thus unchanged. Due to the fact of the set-to-set different locations of the sensor set their presence affects the dynamics of the structure according to mode shape. Sensors located in the nodes of the mode modify the frequency value in minor extent. Models which were obtained from the contactless measurement (model 6 and 9) have higher values of the identified frequencies. Such observation causes a need to assess the statistics of the experimental data sets considering the existence of statistical important differences.

For estimated models tests of t-Student and Wald-Wolfowitz were made for models $3 \mathrm{x}, 3 \mathrm{y}$ and $3 \mathrm{z}$ and also for all pairs for models 4: from set 1 to frame 8 - differences were not significant. Wald - Wolfowitz test was performed 
Table 4

Statistic quantities for the second natural frequency dispersion

\begin{tabular}{ccccc}
\hline Mean value & Median & Minimum & Maximum & Std. Dev. \\
\hline 52,4229 & 52,4260 & 52,1640 & 52,8180 & 0,130612
\end{tabular}

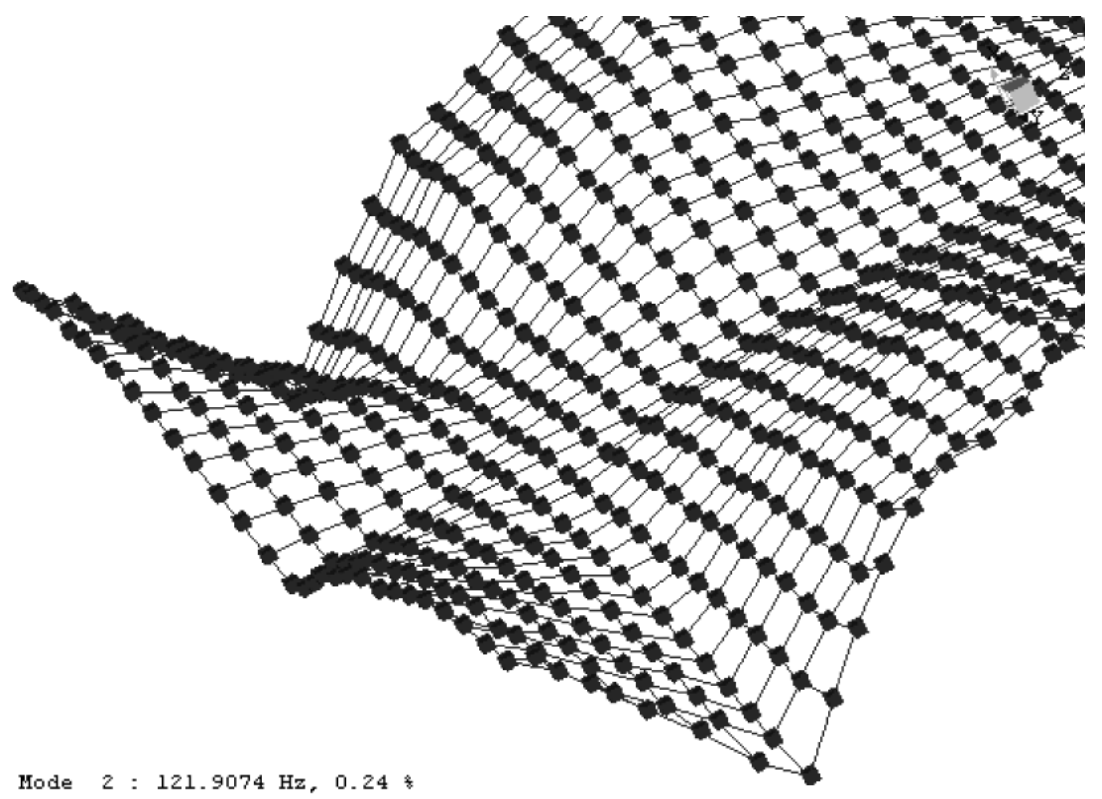

Fig. 4. Plot of the absolute amplitude of the modal displacement.

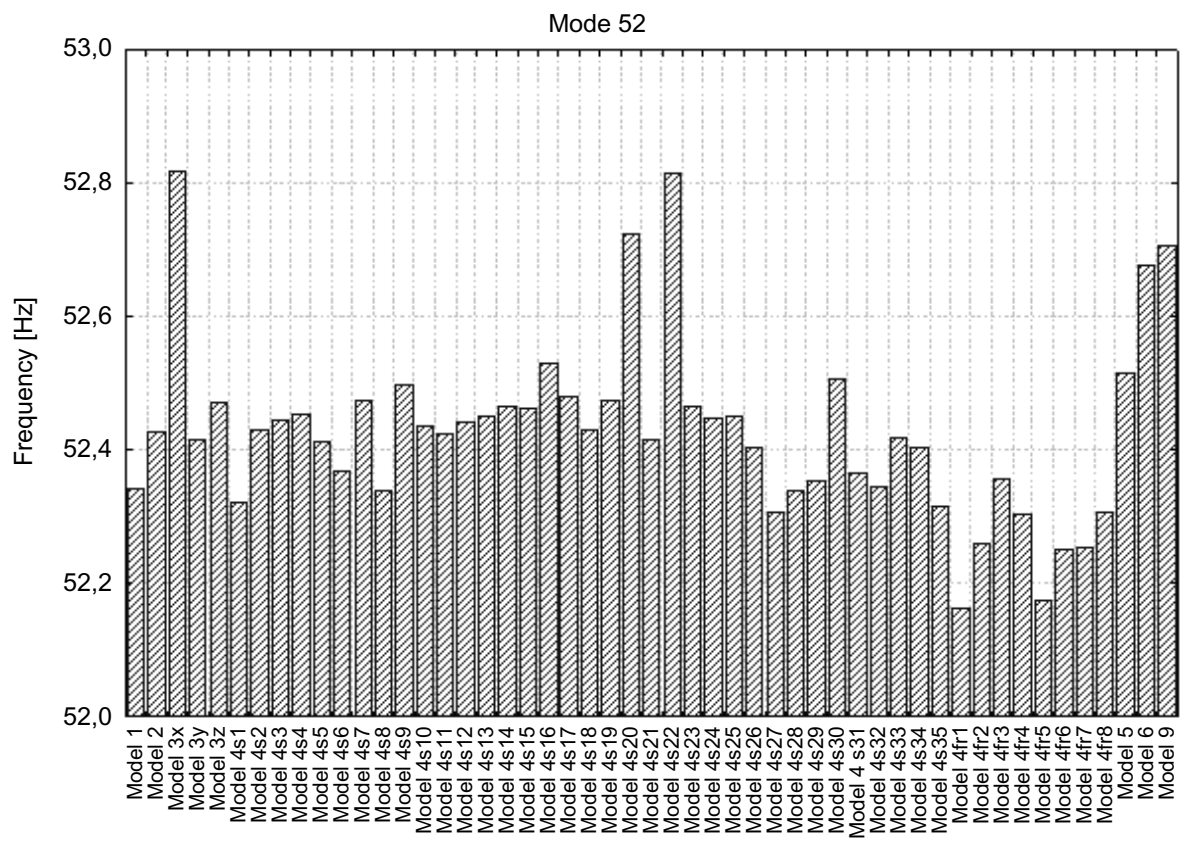

Fig. 5. Measured values of the second natural frequency in first measurement point. 


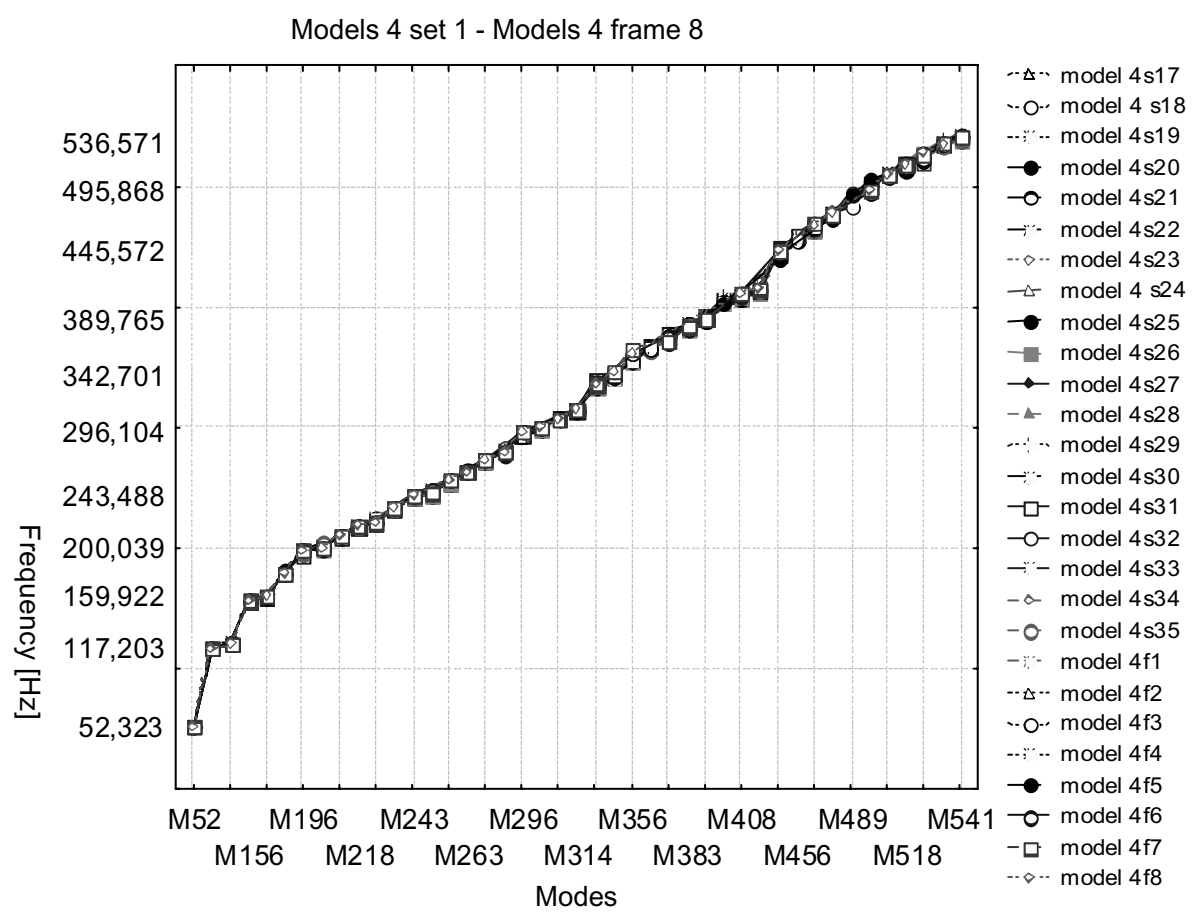

Fig. 6. Frequencies plot for all models estimated on which t-Student tests for models s1-f8 was performed.

to assess if there is normal distribution of experimental data and it's mutually independency. Data which satisfies the assumption of the normal distribution can be a subject of the t-Student test. The t-test is the most commonly used method to evaluate the differences in means between two groups. Figure 6 illustrates data taken into account to tests - it shows that really there are no differences.

For merging the local modal models in multi run modal analysis, natural frequency values obtained from the driving point 1 were chosen due to the fact of their presence in all data sets.

The next step in the statistical assessment of the experimental data set is a correlation analysis of the frequency and damping coefficient for all the modes and all the models. Example for the second natural frequency is presented on the Fig. 7.

Linear correlation coefficient is the measure of the existence (or not) of the relation between two (or more) investigated variables. In statistical analysis the following scale is assumed:

rxy $=-1$ perfect negative correlation

rxy $=0$ variables are not correlated

$0<$ rxy $<0,1$ faint correlation

$0,1<$ rxy $<0,3$ weak correlation

$0,3<$ rxy $<0,5$ average correlation

$0,5<$ rxy $<0,7$ high correlation

$0,7<$ rxy $<0,9$ very high correlation

$0,9<$ rxy $<1$ almost perfect positive correlation

The relationship between frequency and damping is such that as frequency values tend to increase, the damping values tend to decrease. This is represented by a negative correlation presented on the Fig. 7.

In this statistical analysis the test setups were the sources of the variability. Similarly to the procedure Basic statistic quantities were computed. Mean value and standard deviation of the modified frequencies and damping were plotted on Figs 8 and 9. Dimensional frequency modification was done by means of subtracting from a particular mode frequency value its mean value and division by mean value. Similar approach was applied for the damping modification. 
Mode 52

Damping $=24,301-, 4502 *$ Frequency

Correlation coeff.: $r=-, 3431$

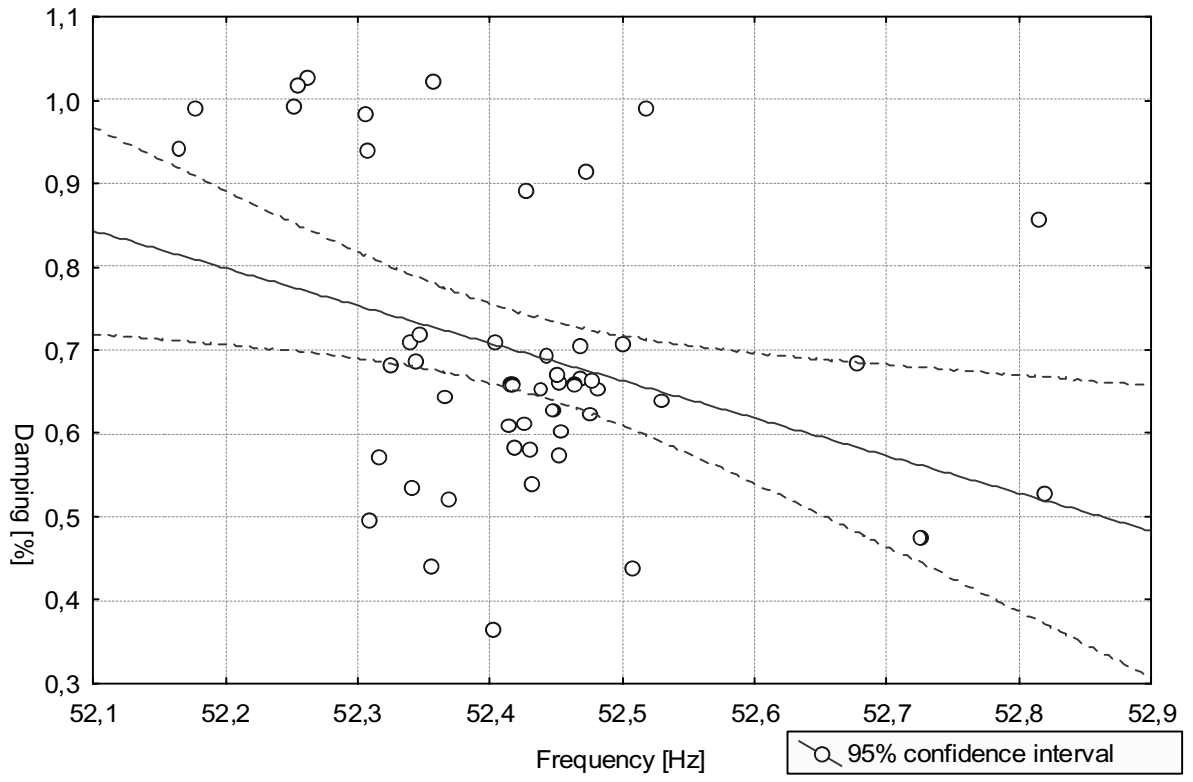

Fig. 7. Plot of the correlation of frequency and damping for second natural frequency.

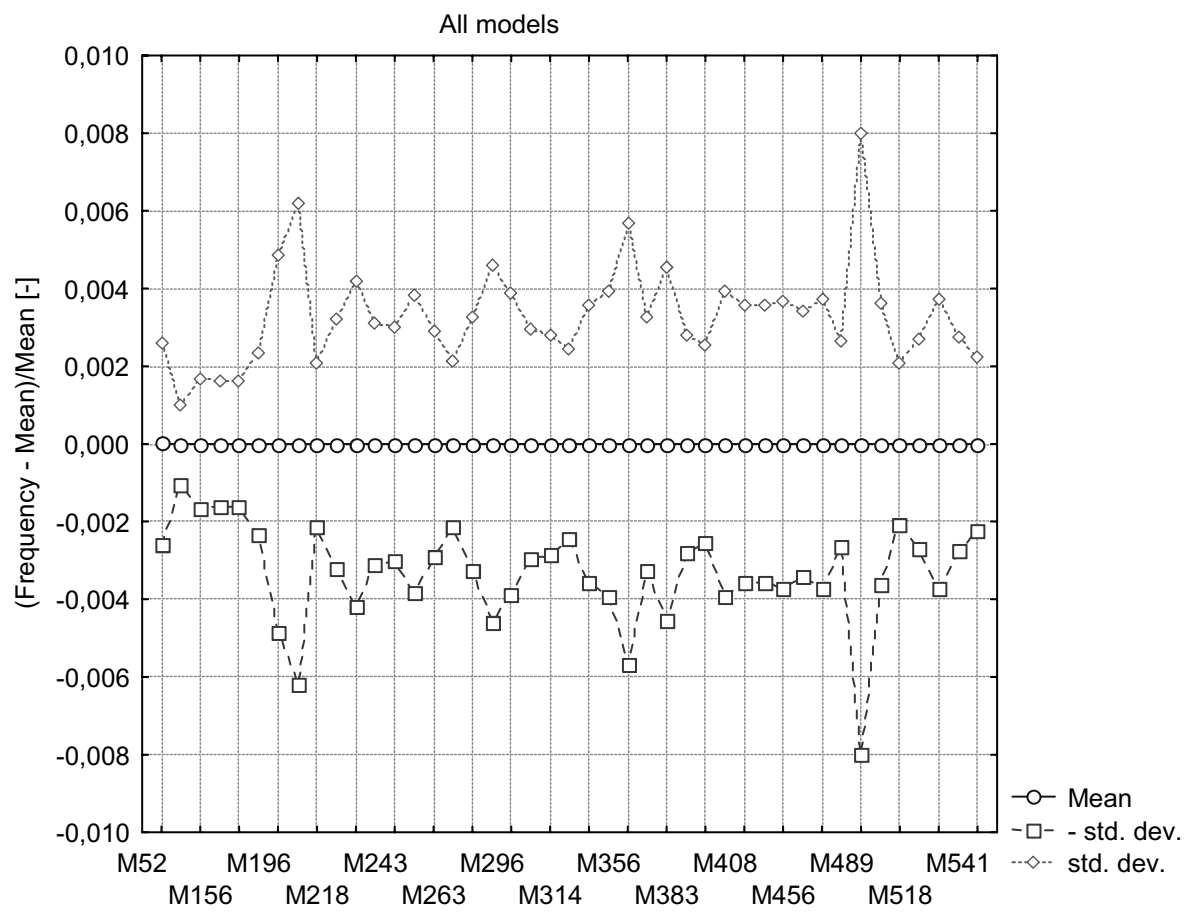

Fig. 8. Plot of the modified frequencies statistic quantities (mean value, std. dev). 


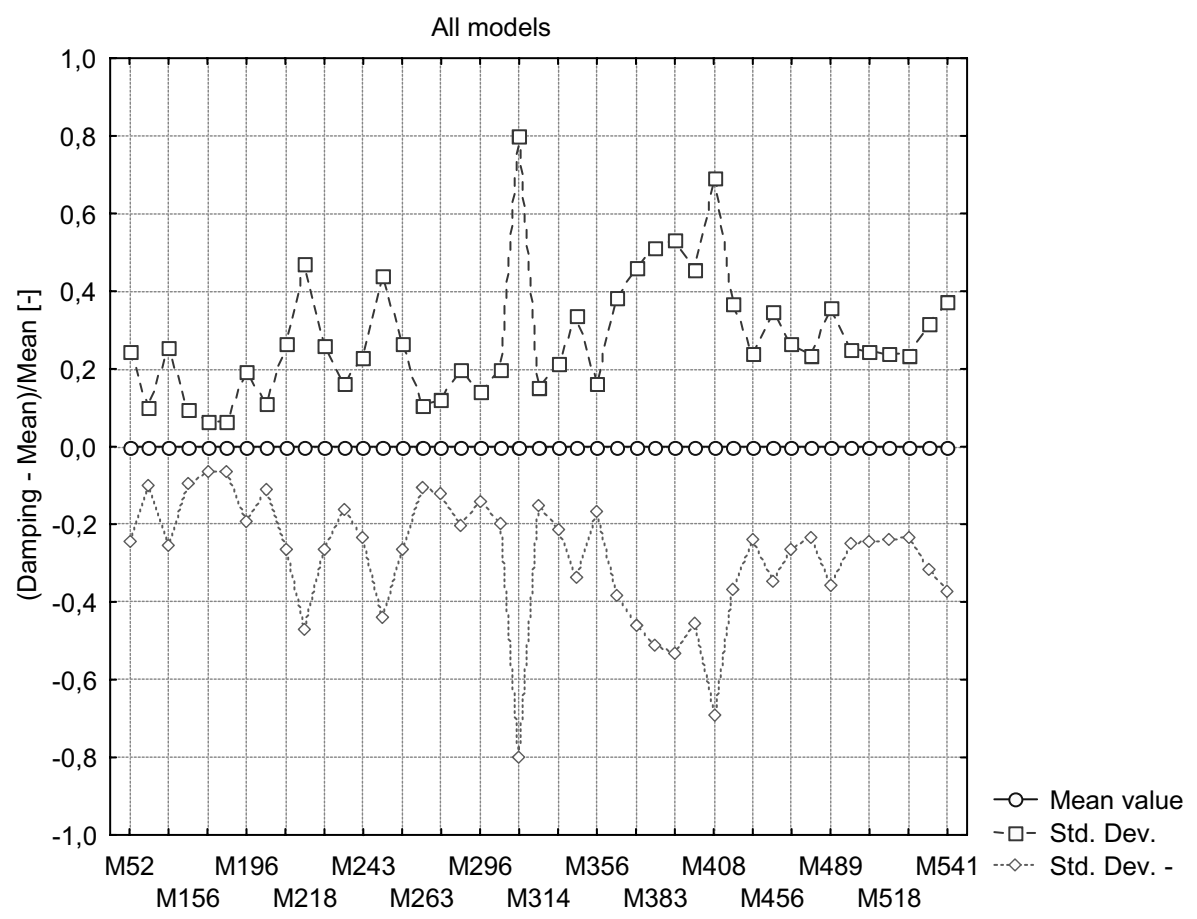

Fig. 9. Plot of the damping ratios statistic quantities (mean value, std. dev).

It is possible to like in similarity concept propose a new approach to assess the variability of modal model parameter as a result of a scatter in measured data. This new concept is based on providing one number which will characterize particular structure in view of its variability and named Variability Level. It is calculated as a mean value of a standard deviation of a modified of frequency and damping.

Modified frequency mean value of std. dev. $=0,003311$

Modified damping mean value of std. dev. $=0,2826$

Calculated values characterize the presence of the test data variability which is reflected in the estimated values of the modal model parameters. Interesting observation from the plots is the increasing standard deviation value towards the higher modes. This can be explained by the fact that the mode shapes become more driven by a local behavior of the structure. The adequate identification of the frequency value in the particular data set becomes more dependent on the sensor placement. Such observation can lead to the conclusion that the measurement point's grid density which is sufficient for successful identification of the low frequency global mode shapes is not necessarily good enough for higher frequency local modes. The evaluation of standard deviation changes within bandwidth of interest can constitute basis of the assessing tradeoff between workload related to application of large number of measurement points and the quality of acquired data.

\section{Finite element modelling and initial updating}

Large panel FE model was created. It consists of the three fundamental parts: skin, frames and stringers. The panel was modeled with two-dimensional shell elements however for the stinger bulb standard BEAM elements were used. The skin has been modeled with three different properties (PCOMP) obtained using different numbers of plies and different sequences of orientation of the plies for each of the three zones indicated in Fig. 10. The first property, in upper panel area is formed by means of 28 plies (14 symmetric), the second property, in lower part, is composed with 14 plies ( 7 symmetric) and third property, in intermediate part, is made up of 15 plies (not symmetric). The thickness of a single ply was set equal to $0.147 \mathrm{~mm}$. Frames, clips and the stingers were modeled with the shell composite orthotropic material. Initial values of the skin characteristics are reported in the Table 5. 
Table 5

Characteristics of single ply of the Prepeg HTA 977-2

\begin{tabular}{lccc}
\hline Parameter & Symbol & Value & Unit \\
\hline Longitudinal Modulus of Elasticity & $\mathrm{E}_{1}$ & $11.35 \mathrm{E} 11$ & $\mathrm{~Pa}$ \\
Lateral Modulus of Elasticity & $\mathrm{E}_{2}$ & $9.3 \mathrm{E} 9$ & $\mathrm{~Pa}$ \\
Poisson ratio & $\nu$ & 0.34 & - \\
Shear Modulus & $\mathrm{G}_{12}$ & $4.88 \mathrm{E} 9$ & $\mathrm{~Pa}$ \\
Density & $\rho$ & 1600 & $\mathrm{~kg} / \mathrm{m}^{3}$ \\
\hline
\end{tabular}

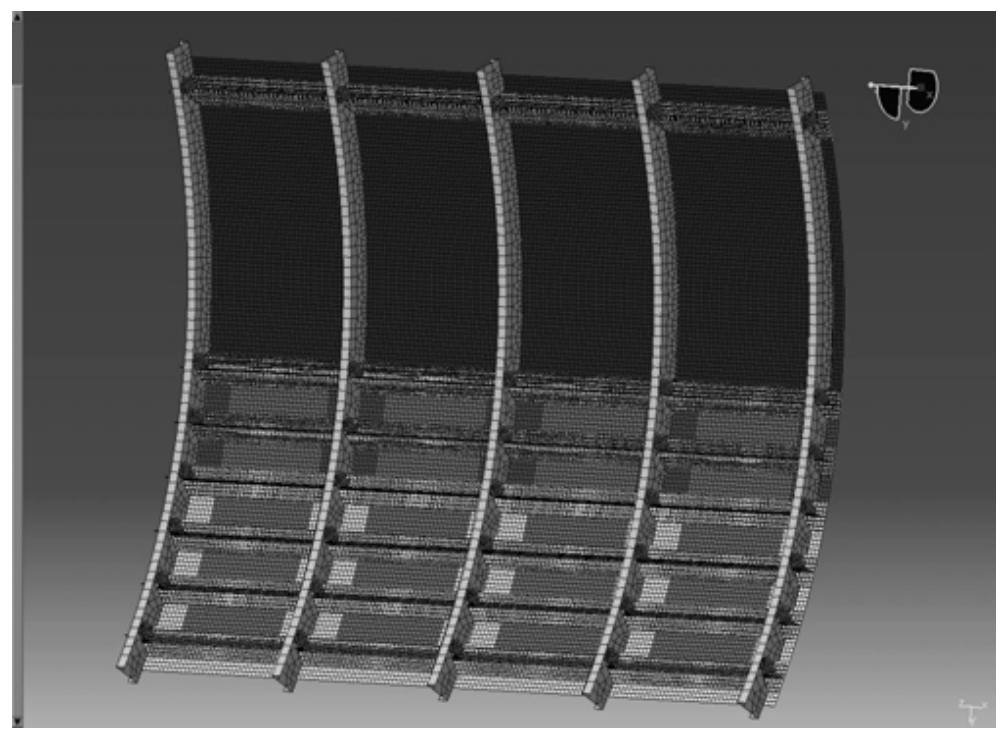

Fig. 10. Finite Element model with panel, frames, stringers, clips and damping layer.
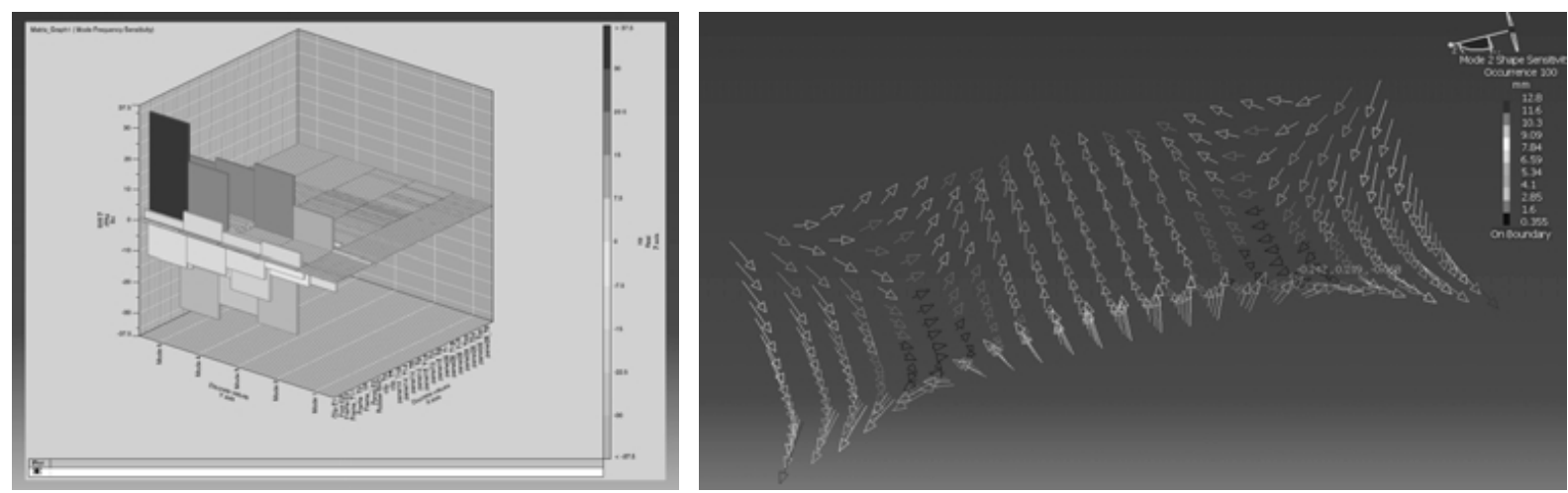

Fig. 11. Frequency (left) and mode shape (right) sensitivity analysis results.

Updating of the panel model according to the experimental results is done by correlation and optimization analysis. In optimization analysis the inverse problem is solved by search for the uncertain model parameters values (like Young modulus, Thickness etc.) which will bring the results best matching the test results. Selection of the model parameters for the input variables in optimization analysis was made based on the results of sensitivity analysis. In the first instance all the model parameters (moduli of elasticity, shear modulus, Poisson's ratio, mass density, ply thickness and fiber orientation) were accounted towards the mode frequency, mode shape and MAC sensitivity analysis (presented on Fig. 11).

Results of the sensitivity analysis clearly indicate that only few model parameters has a significant influence on the 


\begin{tabular}{cccccc}
\multicolumn{7}{c}{ Table 6 } \\
\hline Initial FE [Hz] & TEST M2 [Hz] & Updated FE [Hz] & MAC TEST Updated FE & Test-FE [Hz] & Diff TEST-FE $(\%$ TEST) \\
\hline 3.9 & 4.6 & 3.7 & 0.875 & 0.9 & 24.32432432 \\
60.6 & 52.4 & 50.7 & 0.984 & 1.7 & 3.353057199 \\
129 & 117 & 111.7 & 0.938 & 5.3 & 4.744852283 \\
130.6 & 122 & 119.5 & 0.976 & 2.5 & 2.092050209 \\
146.7 & 156.4 & 138.9 & 0.798 & 17.5 & 12.59899208 \\
148.8 & 159.8 & 141.1 & 0.906 & 18.7 & 13.25301205 \\
181.2 & 180.9 & 163.5 & 0.896 & 0 & 17.4 \\
193.7 & 197.9 & 197.9 & 0.312 & 14.6 & 0 \\
208.7 & 200 & 185.4 & 0.446 & 10.4 & 7.874865156 \\
210.8 & 210.2 & 199.8 & 0.137 & & 5.205205205 \\
\hline
\end{tabular}
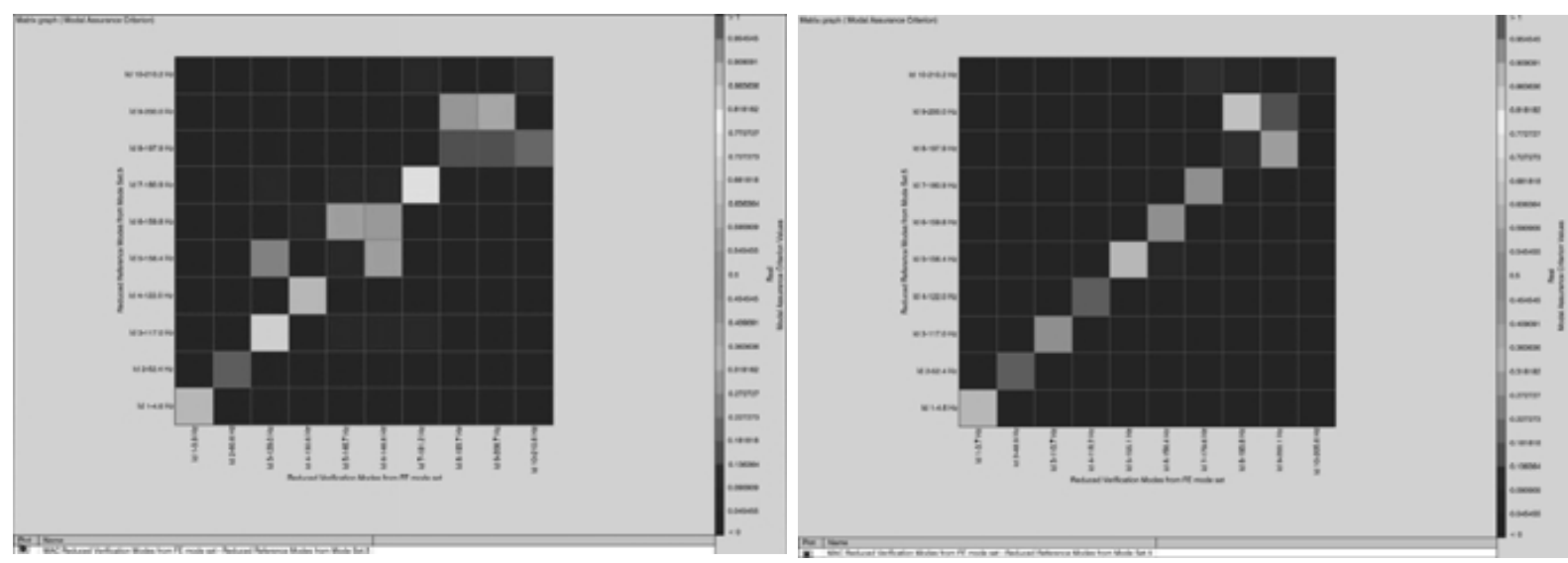

Fig. 12. Left: Initial Test-FE MAC; Right: Updated Test-FE MAC.

considered natural frequencies and mode shapes. For the further updating procedure Young modulus in longitudinal direction density and the orientation of the fibers were selected.. The updating of the FE model was done by means of global optimization with multiobjective criteria. Maximum and minimum values used in the optimization were of $10 \%$ of the nominal model parameters. Criteria defined in the optimization loop were mode shape and frequency values for the nine modes. The updating procedure was repeated for the individual global models. The deterministic values of model parameters most adequately satisfying optimization criteria were found. The final values of the selected model parameters are within initially assumed range of $10 \%$ difference. Table 6 reports examples of the frequency differences between updated model and experimental models

Results assessed by means of the MAC for the initial and updated parameters values are presented in the Fig. 12.

Even if MAC values did not present very high values it has been possible to trace the corresponding mode shape pairs. These mode pair table can be considered close to the optimal correlation line; as a consequence it is possible to assess that within the investigated frequency bandwidth the numerical model presents a dynamical behavior adequately close to the real panel.

\section{Conclusions}

This paper presents some results and aspects of the multidisciplinary and interdisciplinary research oriented for the test data variability and numerical model parameters uncertainties obtained so far within the Unvico-2 project on the large composite fuselage panel structure.

An extensive test campaign performed on the large composite fuselage panel was presented. Test setups included different excitation and measurement techniques of contact and non-contact type. Experimental test data examples were shown and used for modal models estimation, after compared by means of natural frequency, damping ratio and mode shapes. Some general remarks have been formulated. The common observation from displayed comparisons 
is that the accuracy of the results is frequency dependent. The higher the frequency of the mode becomes the larger the discrepancy between models grows. A preliminary FE model of was generated with the aim of predicting the dynamic behavior of the structure in the frequency range corresponding to global modes. It was found that this model was convenient to represent structural behavior but not so surprisingly, was not able to reproduce modal data with accuracy in the higher frequency range. In order to improve the prediction capability beyond the active frequency range, a more detailed model should be first constructed.

\section{Acknowledgements}

Computations were performed on a 50Tflop cluster in TASK Academic Computer Centre in Gdansk, Poland.

This research was supported by a Marie Curie Intra-European Fellowships within the 6th European Community Framework Programme. The authors of this work gratefully acknowledge support for this research under the project No. 038309 "UNVICO-2" provided by the EU.

\section{References}

[1] Special issue (12 papers) of Computer Methods in Applied Mechanics and Engineering devoted to "Computational Methods in Optimization Considering Uncertainties" Volume: 198, Issue: 1 November 15, 2008.

[2] S. Adhikari and C.S. Manohar, Dynamic analysis of framed structures with statistical uncertainties, International Journal for Numerical Methods in Engineering 44(No. 8) (199), 1157-1178.

[3] W.L. Oberkampf, K.V. Diegert, K.F. Alvin and B.M. Rutherford, Variability; uncertainty, and error in computational simulation, The 7th AIAA/ASME Joint Thermophysics and Heat Transfer Conference June 14-18, 1998, Albuquerque, New Mexico.

[4] T.A. Zang, M.J. Hemsch, M.W. Hilburger, S.P. Kenny, J.M. Luckring, P. Maghami, S.L. Padula and W. Jefferson Stroud, "Needs and Opportunities for Risk-Based Multidisciplinary Design Technologies for Vehicles", NASA TM, July 2002.

[5] D.J. Ewins, State-of-the-Art Assessment of Mobility Measurement, a Summary of European Results, Shock and Vibration Bulletin 51 (May 1981).

[6] H. Van der Auweraer, B. Peeters and S. Donders, Importance Of Uncertainty In Identifying And Using Modal Models, Proceedings of the INCE Symposium on Managing Uncertainties in Noise Measurements and Prediction, Le Mans, France: Jun 27-29, 2005.

[7] M.I. Friswell, J.E. Coote, M.J. Terrell, S. Adhikari, J.R. Fonseca and N.A.J. Lieven, Experimental Data for Uncertainty Quantification. 23rd International Modal Analysis Conference, Orlando, Florida, USA, February 2005, paper 201.

[8] K. Mendrok, L. Pieczonka and T. Uhl, Assessment of uncertainty of experimentally obtained modal parameters Proc, ISMA Leuven (B), (2008), 3899-3910.

[9] M. Luczak, A. Vecchio, E. Mucchi, T. Shigeoka and E. Pierro, Experimental Modal Analysis of an Aircraft Fuselage Panel Incorporating Test Data Variabilities, School of Modal Analysis XII Conference, Krakow, 2007.

[10] P.G. Michaelides and S.D. Fassois, Stochastic identification of structural dynamics from multiple experiments - experimental variability analysis, Proc, ISMA Leuven (B), (2008), 3927-3942.

[11] M. Gre'diac, The use of full-field measurement methods in composite material characterization: interest and limitations, Composites: Part A 35 (2004), 751-761.

[12] S. Adhikari and C.S. Manohar, Dynamic analysis of framed structures with statistical uncertainties, International Journal for Numerical Methods in Engineering 44(No. 8) (199), 1157-1178.

[13] M. Shinozuka and F. Yamazaki, Stochastic finite element analysis: an introduction, Stochastic structural dynamics: Progress in theory and applications, edited by S.T. Ariaratnam, G.I. Schueller and I. Elishakoff, Elsevier Applied Science, London, 1998.

[14] I. Elishakoff and Y.J. Ren, Large Variation "Finite Element Method for Stochastic Problems", Oxford University Press, Oxford, U.K., 2003.

[15] M. Hanss, The transformation method for the simulation and analysis of systems with uncertain parameters, Fuzzy Sets and Systems 130(Issue 3) (16 September 2002), 277-289.

[16] S. Rao and P. Sawyer, Fuzzy finite element approach for the analysis of imprecisely defined systems, AIAA J 33(12) (1995), $2364-2370$.

[17] S. Donders, van de peer, Joost, S. Dom, H. Van der Auweraer and D. Vandepitte, Parameter Uncertainty and Variability in the Structural Dynamics Modeling Process, Proceedings of the IMAC XXII Dearborn USA, Jan 26-29, 2004.

[18] M.S. Kompella and R.J. Bernhard, Measurement of the Statistical Variation of Structural-Acoustic Characteristics of Automotive Vehicles, Noise Control Engineering Journal 44(2) (March-April 1996), 93-99.

[19] H. Van der Auweraer, W. Leurs, P. Mas and L. Hermans, Modal Parameter Estimation from Inconsistent Data Sets, Proc. 18th IMAC, (Feb 7-10 2000), San Antonio (TX-US), pp. 763-771.

[20] B. Peeters, H. Van Der Auweraer, P. Guillaume and J. Leuridan, The Polymax Frequency-Domain Method: a New Standard for Modal Parameter Estimation? Shock And Vibration, Special Issue dedicated to Professor Bruno Piombo 11 (2004), 395-409.

[21] M. Friswell and J. Mottershead, Finite Element Model Updating in Structural Dynamics, Kluwer Academic Publishers, Dordrecht, 1995.

[22] N. Maia and J. Silva, Theoretical and Experimental Modal Analysis, Editors Maia \& Silva, Published by Research Studies Press, Distributed by John Wiley Sons, May 1997. 
[23] E. Dascotte, The use of FE model updating and probabilistic analysis for dealing with uncertainty in structural dynamics simulation, Japan Modal Analysis Conference (Sep 10-12 2003), Tokyo, Japan.

[24] E. Capiez-Lernout, C. Soize and R. Ohayon, Robust parametric updating of uncertain finite element models from experimental modal analysis, Proc ISMA (2008) Leuven (B), 1839-1854.

[25] H. Haddad Khodaparast and J.E. Mottershead, Efficient Methods in Stochastic Model Updating, Proc ISMA (2008) Leuven (B), 1855-1870.

[26] E. Zhang, J. Antoni and P. Feissel, Bayesian identification and updating of uncertain modal parameters in the frequency domain, Proc ISMA 2008, Leuven (B), 3975-3984.

[27] M. Luczak, A. Vecchio, B. Peeters and E. Pierro, Contact versus Non-contact measurement of a Large Composite Fuselage Panel. 

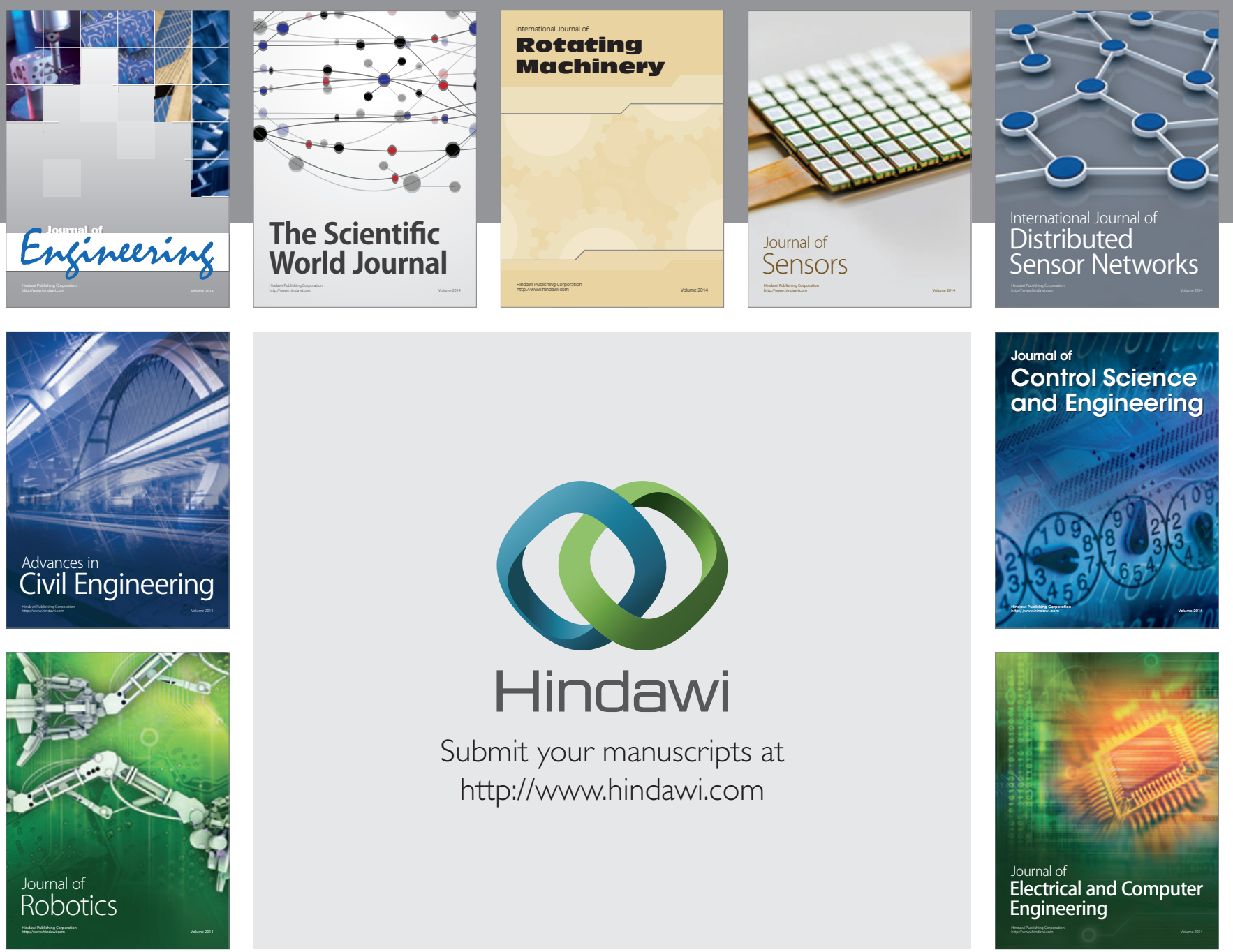

Submit your manuscripts at

http://www.hindawi.com
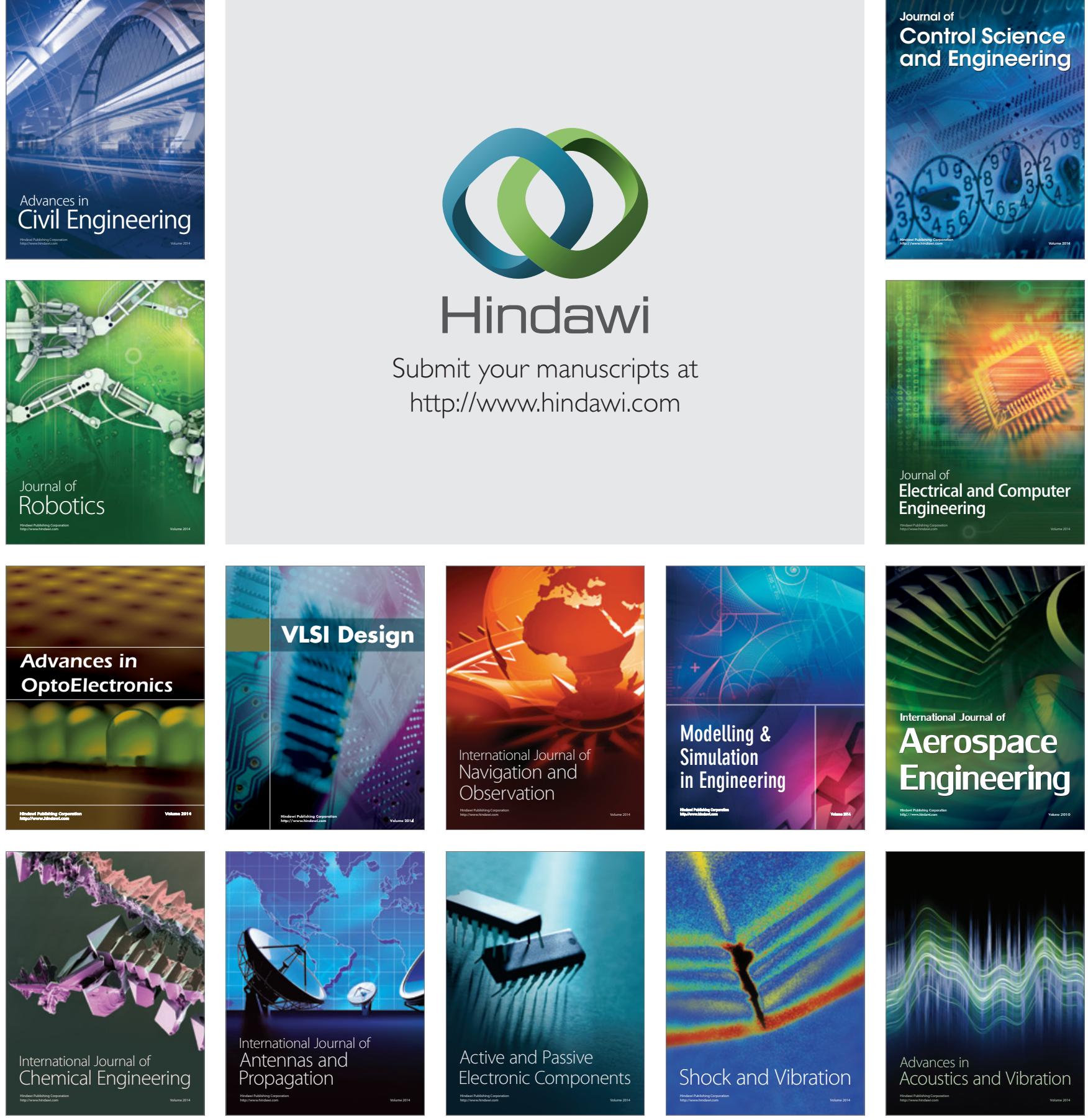